\title{
PROBABILISTIC SEISMIC HAZARD ANALYSIS OF NEPAL
}

\author{
Sunita Ghimire*1
}

\begin{abstract}
Probabilistic seismic hazard analysis for Nepal has been carried out considering uniform density model. A detailed earthquake catalogue since 1255 A.D, within the rectangular area has been developed and historical earthquakes are plotted in the map of Nepal. Five hundred twenty eight numbers of areal sources are used within the study area to characterize the seismic sources. The completeness of the data has been checked by using Stepp's procedure. Seismicity in four regions of study area has been evaluated by defining 'a' and 'b' parameters of Gutenberg Richter recurrence relationship. Seismic hazard curve of Nepal for soft subsoil condition for $\mathbf{1 0 \%}$ probability of exceedence in $\mathbf{5 0}$ years period i.e. for return period of $\mathbf{4 7 5}$ years has been plotted.
\end{abstract}

Keywords: PSHA - Seismic Hazard Curve - Attenuation relationship

\section{INTRODUCTION}

Nepal is highly susceptible to earthquake related hazards like ground shaking, structural damage and destruction, liquefaction, landslide, flood, lifeline damage and obstruction etc. Recent Gorkha earthquake on 25th April 2015 of magnitude 7.8M with another strong aftershock of magnitude $7.3 \mathrm{M}$ caused about 9000 deaths, 22000 injuries with loss of billions of dollars is one of the clear example of devastation during the hazard. To mitigate such hazard, there is not any other alternative left for professionals rather than making the structures earthquake resistant. For the design of seismic resistant structures, it is essential to do site specific seismic hazard analysis and to quantify the site specific ground motion parameters. This necessitates the probabilistic seismic hazard analysis for the whole country. So an attempt has been made to carry out probabilistic seismic hazard analysis of the country and the result is presented in the form of contour map for PGA and spectral accelerations.

\section{Earthquake Catalogue}

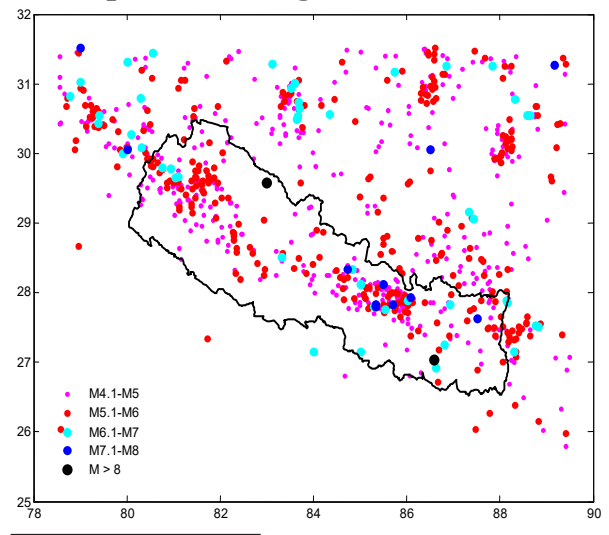

Fig 1: Earthquake density in study area Earthquake catalogue was obtained by merging data from U.S. Geological Survey, National earthquake Information Centre (NEIC), Rana 1935, BECA 1993, Pant 2000, Ambraseys and Douglas 2004, Ambraseys N. and Jackson D. 2003, and Lave et.al (2005). To achieve uniformity in the data, all the magnitudes or intensities are converted to moment magnitude (Hank

Department of Civil Engineering, IOE Thapathali Campus, Tribhuvan University, Nepal

Corresponding author

Email: ghimiresunitag@gmail.com 
and Kanamori 1979) using various relationships (McGuire 2004) and scaling relationship for Himalayan region (Ambraseys and Douglas 2004). The earthquake record contains large number of aftershocks which if not removed leads the earthquake data to be a non Poissoinian. This makes the statistical analysis more complicated. Hence the aftershocks are removed based on windowing algorithm given by Gardner and Knopoff 1974.For this the aftershocks are identified based on its distance from epicenter of main shock and time difference in occurrence with main shock. The catalogue after the aftershock removal follows Poissoinian distribution. There are total 1228 records available among them 827 events are found to be the main events.

\section{Completeness Analysis}

It is very difficult to allocate the location of earthquake occurrence and more than this it is difficult to specify which earthquake belongs to which fault. The recorded earthquake data has non uniformity in its number due to the difficulty in availability of data of old times. Hence it is necessary to do the completeness analysis for the best fit of frequency formula and it can be done as per Stepp 1972. For the earthquakes events are grouped into small intervals of time and each magnitude range $(0.5 \mathrm{M})$ is judged separately. If $k_{1}, k_{2}$, $k_{3} \ldots k_{n}$ are the number of earthquakes per unit time interval, then an unbiased estimate of the mean rate per unit time interval of the sample is

$$
\lambda_{m}=\frac{1}{n} \sum_{i=1}^{n} k_{i}
$$

The variance of the sample is given by,

$$
\sigma_{\lambda_{m}}^{2}=\frac{\lambda_{m}}{n}
$$

Equation 2

Where,

$\mathrm{n}=$ number of unit time intervals

If we assume number of unit time intervals as 1 year then standard deviation of the estimate of the mean $\sigma_{\lambda_{m}}=\frac{\sqrt{\lambda_{m}}}{\sqrt{T}}$

Where, $\mathrm{T}=$ sample length
Thus, assuming stationarity, we expect that $\sigma_{\lambda_{m}}$ behaves as $\frac{1}{\sqrt{T}}$ in the subinterval of the sample in which the mean rate of occurrence $\frac{N}{T}$ in each magnitude class constant.

Where, $\mathrm{N}=$ cumulative number of earthquakes in the time interval $\mathrm{T}$

The completeness analysis as done above gives the best fit for the magnitude frequency relation. In this case the magnitude frequency relationship for all the four areas of Nepal is obtained. 


\section{Probabilistic Seismic Hazard Analysis (PSHA) Spatial Uncertainty}

Due to uncertainty in location and nature (geometry) of the source it is very much difficult to define the source zone. In this study, the area is divided into 528 numbers of smaller areal elements of size $0.5^{\circ}$ along longitude and $0.25^{\circ}$ along latitude. All the sources are assumed to be equally capable of producing earthquake and the occurrence will be in the centre of each areal cell.

\section{Magnitude Uncertainty}

To address the uncertainty in magnitude produced by each source zone various recurrence relationships specifying the average rate at which an earthquake of some size will be exceeded is to be developed. Thus obtained magnitude frequency relationship may accommodate the maximum size earthquake. The recurrence relation as per Gutenberg and Richter is,

$$
\begin{array}{ll}
\log \lambda_{m}=a-b M & \text { Equation } 4
\end{array}
$$

Where,

$\lambda_{m}=$ mean annual rate of exceedence of magnitude $\mathrm{M}$

$10^{\mathrm{a}}=$ mean yearly number of earthquakes of magnitude greater than or equal to zero

$\mathrm{b}=$ relative likelihood of large and small earthquakes

As $b$ value increases the number of larger magnitude earthquake decreases compared to those of smaller magnitude earthquakes.

The standard Gutenberg-Richter law predicts the non-zero mean rate of exceedences for magnitudes up to infinity. We are concerned with the earthquake greater than magnitude M4.5 since greater size earthquake produces maximum level of shaking generally. So bounded recurrence relation law is used to express the certain maximum magnitude $\mathrm{M}_{\max }$ associated with each source zone the value of which is greater than minimum magnitude $\mathrm{M}_{\text {min }}$. The probability density function for Gutenberg Richter law with lower and upper bound magnitude is given by

$$
f_{M}(m)=\frac{\beta \exp \left[-\beta\left(m-m_{\min }\right)\right]}{1-\exp \left[-\beta\left(m_{\max }-m_{\min }\right)\right]}
$$


Where, $\beta=2.303 \mathrm{~b}$

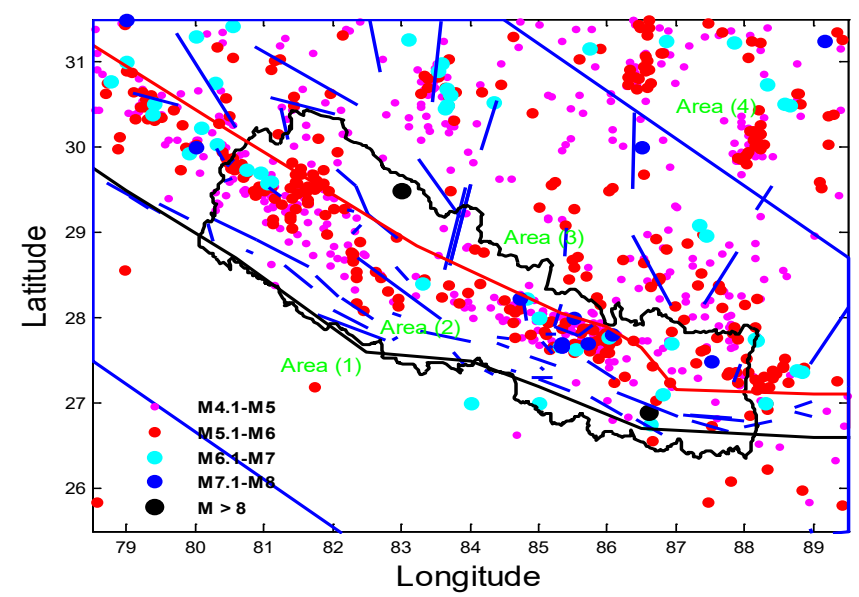

Fig 2: Delineation of seismic source zones

The areal seismicity in this study is presented in this table.

Table 1: Seismicity distribution in the area

\begin{tabular}{|c|c|c|c|}
\hline Area & $\mathbf{a}$ & $\mathbf{b}$ & $\mathbf{M}_{\max }($ year $)$ \\
\hline$; 1 ; \mathrm{p} 1$ & 5.18 & -1.27 & $6.6(1833)$ \\
\hline 2 & 5.04 & -0.91 & $8.1(1934)$ \\
\hline 3 & 6.3 & -1.16 & $8.2(1505)$ \\
\hline 4 & 5.86 & -1.18 & $7.2(1934)$ \\
\hline
\end{tabular}

\section{Temporal uncertainty}

of occurrences of a particular event during a given time interval is given by

$$
p[N=n]=\frac{\mu^{n} e^{-\mu}}{n !}
$$

Equation 6

Where, $\mu=$ average number of occurrences of the event at the given time interval

So for seismic hazard assessment purposes,

$$
p[N=n]=\frac{(\lambda t)^{n} e^{-\lambda t}}{n !} \quad \text { Equation } 7
$$

Where,

$\lambda=$ average rate of occurrence of the event. $\quad \mathrm{t}=$ time period (years)

The probability of occurrence of at least one event in time period $\mathrm{t}$ is given by $p[N=n]=p[N=1]+p[N=2]+.$.

$=1-e^{-\lambda t}$

Then at least one exceedence of particular magnitude in a period of years $t$ is written as 
$p[N \geq 1]=1-e^{-\lambda t}$

Equation 8

Similarly, probability of exceedence of particular parameter $y^{*}$ in a time period $t$ is given by

$p\left[Y \geq Y^{*}\right]=1-e^{-\lambda_{Y} \cdot t}$

Equation 9

Where, the return period o of $\mathrm{y}^{*}$ is defined as:

$R_{y^{*}}\left(y^{*}\right)=\frac{1}{\lambda\left[Y \geq y^{*}\right]}=\frac{-t}{\ln \left(1-p\left(\left[Y \geq y^{*}\right]\right)\right.}$

Equation 10

\section{Attenuation of ground motion}

The seismic hazard at any area depends upon the attenuation characteristics of that site, which is the function of magnitude of earthquake, source to site distance and geologic characteristics of the site or tectonic environment. Proper implementation of most modern ground motion attenuation relationship requires that the seismic sources are characterized by the details of the fault - rupture model. There are the various attenuation relationships developed by researchers at different site condition. As Nepal lies in the subduction zone, the attenuation laws developed for subduction zone is used to to develop the peak ground acceleration (PGA) and spectral acceleration (SA). So, three attenuation relationships developed for subduction zone have been used here and mean of them is used to calculate PGA.

1. Young's et al 1997

$$
\begin{aligned}
& \ln (Y)=0.2418+1.414 M+C_{1}+C_{2}(10-M)^{3}+ \\
& C_{3} \ln \left(r_{r u p}+1.7818 e^{0.554 M}\right)+0.00607 H+0.3846 Z_{T}
\end{aligned}
$$

Equation 11

Standard deviation $=C_{4}+C_{5} M$

Where,

$\mathrm{Y}=$ spectral acceleration

$\mathrm{M}=$ Moment magnitude

$r_{\text {rup }}=$ source to site distance $(\mathrm{km})$

$\mathrm{H}=$ focal depth $(\mathrm{km})$

$C_{k, k=1 t o 10}=$ coefficients determined by regression analysis

$\mathrm{Z}_{\mathrm{T}}=$ source type, ( 0 for interface and 1 for intra slab)

2. Kanno et. al. (2006)

$$
\begin{aligned}
& \log (\text { pre })=a_{1} M_{W}+b_{1} X-\log (X+ \\
& \left.d_{1} 10^{0.5 M_{w}}\right)+c_{1} \\
& \text { Where, pre }=\text { in } \mathrm{cm} / \mathrm{s}^{2} \\
& a_{1}, b_{1}, c_{1}, d_{1}=\text { constants having values } 0.56,-0.0031,0.26,0.0055 \text { respectively } 12
\end{aligned}
$$

3. Zhao et. al. (2006) Zhao et. al. (2006) $\begin{gathered}\log _{10} P G A=A_{1} M_{w}+A_{2} \log _{10} \sqrt{r^{2}+d^{2}} \\ +A_{3} h_{c}+A_{4}+A_{5} \delta_{R}+A_{6} \delta_{A}+A_{7} \delta_{I}\end{gathered} \quad$ Equation 13

Where, PGA $=$ Peak ground acceleration $\left(\mathrm{m} / \mathrm{s}^{2}\right)$

The terms containing $\delta$ depends on soil types.

The terms containing A are constants. 


\section{Seismic Hazard Curve}

The plot of mean annual rate of exceedence versus peak ground acceleration gives the seismic hazard curve. The seismic hazard curve for individual source zone is obtained at first and they are combined to get the hazard for the particular site. . The probability of exceedence of certain ground motion is estimated by assuming probability distribution of ground motion. The probability of exceedence of certain ground motion parameter $\mathrm{Y}$ than the particular value $\mathrm{y}^{*}$ is calculated for one possible earthquake at one possible source location is multiplied by the probability that the particular magnitude earthquake will occur at the particular location. This process is repeated for all possible magnitudes and locations with the probabilities of each summed. For a given earthquake occurrence, the probability that a ground motion parameter $\mathrm{Y}$ will exceed particular value $\mathrm{y}^{*}$ can be computed by using total probability theorem. i.e.

$$
\begin{array}{ll}
p\left[Y>y^{*}\right]=p\left[Y>y^{*}|X| p[X]\right. & \text { Equation } 14 \\
=\int p\left[Y>y^{*}|X| f_{x}(X) d x\right. &
\end{array}
$$

Where, $\mathrm{X}=$ a vector of random variable that influences $\mathrm{Y}$. In most cases quantities in term $\mathrm{X}$ are limited to the magnitude $\mathrm{M}$ and distance $\mathrm{R}$. Assuming $\mathrm{M}$ and $\mathrm{R}$ are independent, the probability of exceedence can be written as

$p\left[Y>y^{*}\right]=\iint p\left[Y>y^{*} \mid m, r\right] f_{M}(m) f_{R}(r) d m d r$

Equation 15

Where,

$p\left[Y>y^{*} \mid m, r\right]=$ obtained from predictive relationship

$f_{M}(m)=$ probability density function of magnitude

$f_{R}(r)=$ probability density function of distance

If the site of interest in a region of $\mathrm{N}_{\mathrm{s}}$ potential earthquake sources each of which has an average rate of threshold magnitude exceedence, $v_{i M}=\exp \left(\alpha-\beta M_{\min }\right)$, the total average rate of exceedence for the region will be given by

$\lambda_{y^{*}}=\sum_{i=1}^{N_{s}} v_{i} \iint P\left[Y>y^{*} \mid m, r\right] f_{M}(m) f_{R}(r) d m d r \quad$ Equation 16

Since the individual terms of equation 16 are difficult to obtain analytically by integration, the possible magnitudes and distances and divided into $N_{M}$ and $N_{R}$ segments respectively. Then mean rate of exceedence can be obtained by

$$
\begin{array}{r}
\lambda_{y^{*}}=\sum_{i=1}^{N_{s}} \sum_{j=1}^{N_{M}} \sum_{k=1}^{N_{R}}\left[v_{i} P\left[Y>y^{*} \mid m_{j}, r_{k}\right]\right. \\
\left.f_{M}\left(m_{j}\right) f_{R}\left(r_{k}\right) \Delta m \Delta r\right]
\end{array}
$$

Where, $m_{j}=m_{\min }+(j-0.5)\left(m_{\max }-m_{\min }\right) / N_{M}$

$$
r_{k}=r_{\min }+(k-0.5)\left(r_{\max }-r_{\min }\right) / N_{R}
$$




$$
\begin{aligned}
& \Delta m=\left(m_{\max }-m_{\text {min }}\right) / N_{m} \\
& \Delta r=\left(r_{\text {max }}-r_{\text {min }}\right) / N_{R}
\end{aligned}
$$

This is equivalent to assuming that each source is capable of generating only $\mathrm{N}_{\mathrm{M}}$ different earthquakes of magnitude, $m_{j}$, at only $N_{R}$ different source to site distances, $r_{k}$ Equation 17 is then equivalent to

$$
\begin{aligned}
& \lambda_{y^{*}}=\sum_{i=1}^{N_{S}} \sum_{j=1}^{N_{M}} \sum_{k=1}^{N_{R}} v_{i} P\left[Y>y^{*} \mid m_{j}, r_{k}\right] \quad \text { Equation } 18 \\
& p\left[M=m_{j}\right] p\left[R=r_{k}\right]
\end{aligned}
$$

\section{Uniform Density Model}

As it is explained in previous sections, seismic hazard from faults cannot be estimated as it is done in conventional methods. If there is the lack of recognizable earthquake faults and seismically active geologic structure in any area then uniform density model is adopted. In such model the earthquake densities are equally distributed in all areas weather there is earthquake or not. Maximum magnitudes for these area sources are typically assessed from an extrapolation of historical seismicity of the region, from compelling worldwide analogs of the regional tectonic setting from regional paleoseismologic data and interpretations (if available), or simply from the judgments of experts. Uniform density model forgets faulting and assumes uniform geology and gives the equal weightage to all the area capable of producing earthquake.

\section{Results and discussions}

Following the above mentioned theory and procedures and by using code in Matlab, obtained results are presented here.

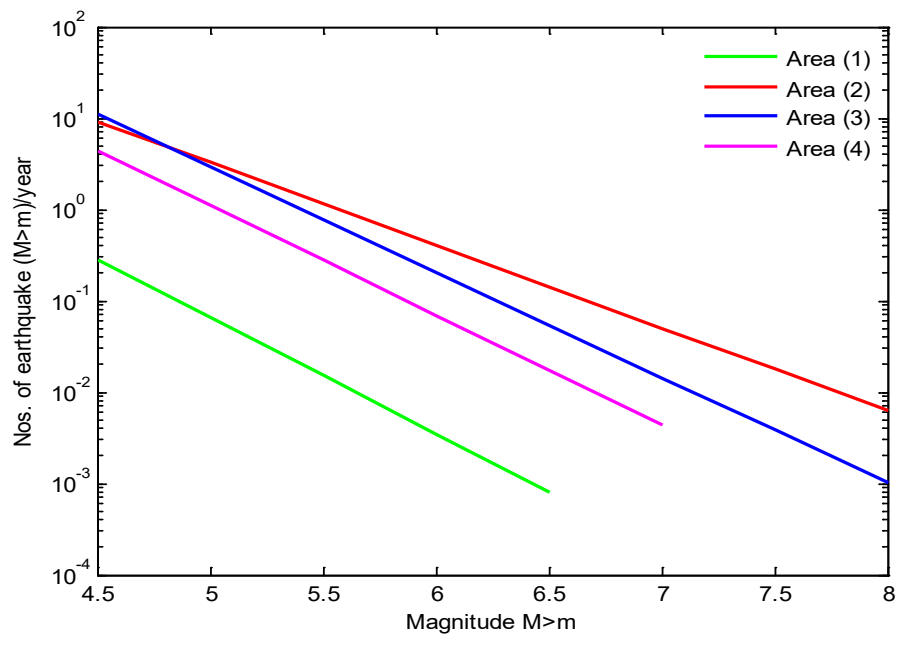

Fig 3: Magnitude frequency relationship 
The curve with highest slope of area 1 indicates that there is lacking of major earthquake in this region and the flattest slope in area 2 indicates there is the major earthquake in the region. When the database is complete, the rate will be nearly constant. ${ }^{2}$

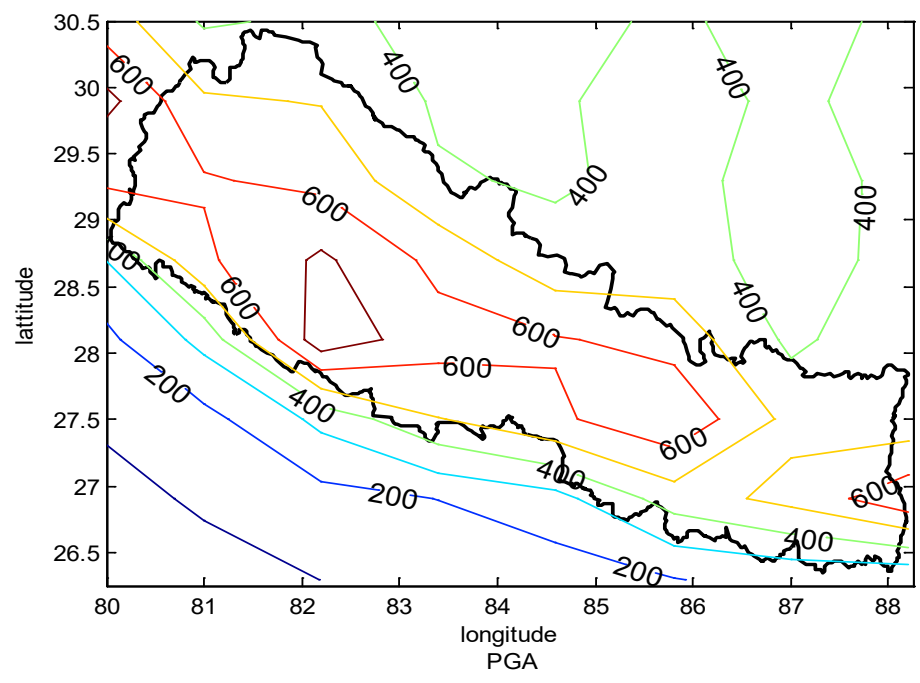

Fig 5: PGA with return period of 475 years on soft soil (5\% damping)

2 BECA WORLEY INTERNATIONAL. Seismic Hazard Mapping and Risk Assessment for Nepal. New Zealand, 1993.

Gardner J.K. and Knopoff L. "Sequence of earthquake in southern California with aftershock removal." Bulletin of the seismological society of America (October 1974): 16(5).

Gutenberg, B. and Richter, C.F. "Frequency of Earthquakes in California." Bulletin of Seismological Society of America 34.4 (1944): 1985-1988.

Kanno, T., Narita, A., Morikawa, N., Fujiwara, H., and Fukushima, Y. "A new attenuation relation for strong ground motion in Japan based on recorded data." Bulletin of Seismological Society of America 96.3 (2006): 879-897.

Kanno, T., Narita, A., Morikawa, N.,Fujiwara, H. and Fukushima, Y. "ANnew Attenuation Relation for Strong Ground Motion in Japan based on Recorded Data." Bulletin of Seismological Society of America 96.3 (2006): 879-897.

Kramer, S.L. "Geotechnical earthquake engineering." Prentice-Hall International series in Civil Engineering and Engineering Mechanics (1996).

Mcguire, R. K. "Seismic hazard and risk analysis." Earthquake Engineering Research Institute, MNO-10 (2004).

Pandey, M. R. "A step towards a historical seismicity of Nepal." Fransco-Nepalese conference on peoples, environment, and landscapes of Himalayas. (2000).

Pandey, M. R., and Molnar, P. "The distribution of intensity of Bihar-Nepal earthquake 15 January 1934 and bounds on the extent of the rupture zone." Journal of Nepal Geological Society 5.1 (1988): 22-44.

Pandey,M.R. , Chitrakar,G.R. , Kafle, B.,Sapkota, S.N. , Rajaure, S. and Gautam,U.P. Seismic Hazard Map of Nepal. Kathmandu, Nepal, 2002.

Parajuli, H.R. "Seismic Hazard Analysis of Nepal, an application to Nepal, considering area sources." (2010).

Parajuli, H.R., Kiyono, J.,Taniguchi, H. and Maskey, P.N. "Probabilistic Seismic Hazard Assessment for Nepal." (2008).

Rana B. S. J. R. Nepal's Great Earthquake 1934 (in Nepali). Sahayogi Press, Tripureshwor, Kathmandu, 1935.

Stepp, J.C. "Analysis of completeness of the earthquake sample in the Pudet Sound area and its effect on statistical estimates of earthquake hazard." Proceedings of the first microzonation conference. Seattle, WA, 1992. 897-909. 